CORRESPONDENCE

\title{
Repurposing antidepressants inhibiting the sphingomyelinase acid/ceramide system against COVID-19: current evidence and potential mechanisms
}

(c) The Author(s), under exclusive licence to Springer Nature Limited 2021

Molecular Psychiatry (2021) 26:7098-7099; https://doi.org/10.1038/s41380021-01254-3

\section{TO THE EDITOR:}

We read with great interest the correspondence letters of Salles et al. [1] and Stip et al. [2], following our multicenter observational retrospective study that showed a substantial association between antidepressant use and reduced risk of intubation or death in 7230 patients hospitalized for COVID-19 [3]. Salles et al. suggest that combining an antidepressant such as fluoxetine with rimonabant, an inverse agonist of CB1 cannabinoid receptor, may be useful against COVID-19, thanks to the antiviral and anti-inflammatory effects of the former and the potentially complementary antiinflammatory properties of the later. Stip et al. significantly summarized the growing body of evidence of the potential benefit of different psychotropic medications in COVID-19 and their possible underlying mechanisms. They suggested further elucidation of ways that certain antidepressants may be acting in this indication. These two letters challenge us on the potential mechanisms that may underlie the potential positive effect of certain antidepressants on the course of COVID-19. This knowledge is crucial to help identify the more promising molecules for COVID-19 and help design trials evaluating these molecules.

Since the initial release of our results in July, 2020 [4], several important studies have led to a substantially improved understanding of the mechanisms that may underlie the potential positive effect of certain antidepressants.

First, molecules such as fluoxetine, fluvoxamine, paroxetine, escitalopram, or amitriptyline are antidepressants that belong to the group of functional inhibitors of acid sphingomyelinase (ASM), called FIASMA [5-7], that also comprises other medications commonly used in clinical practice, such as antihistamine medications (e.g., hydroxyzine, promethazine), calcium channel blockers (e.g., amlodipine, bepridil), and mucolytics (e.g., ambroxol [7]). These pharmacological compounds in vitro and in vivo inhibit ASM, an enzyme that catalyzes the hydrolysis of sphingomyelin into ceramide and phosphorylcholine [5-7]. Preclinical evidence indicates that SARS-CoV-2 activates the ASM/ceramide system, resulting in the formation of ceramide-enriched membrane domains that facilitate viral entry and infection by clustering ACE2, the cellular receptor of SARS-CoV-2 $[6,7]$. The inhibition of the ASM/ceramide system by FIASMA antidepressants prevented infection of Vero E6 cells with SARS-CoV-2. Importantly, the reconstitution of ceramides in cells treated with these antidepressants restored the infection [6]. In healthy volunteers, oral administration of a low dose of the FIASMA antidepressant amitriptyline prevented infection of freshly isolated nasal epithelial cells with SARS-CoV-2 spike protein pseudotyped particles within $2 \mathrm{~h}$, which was also restored after the reconstitution of ceramides in these cells [6]. These preclinical data were confirmed by another study that demonstrated an inhibition by fluoxetine of SARS-CoV-2 infection in cultured epithelial cells [8]. The potential benefit of FIASMA treatments among patients hospitalized for severe COVID-19 was recently explored in an observational multicenter retrospective study [9]. Therein, it was reported that taking a FIASMA medication upon hospital admission was associated with substantially reduced likelihood of intubation or death. This association was not specific to one FIASMA class (e.g., FIASMA antidepressants) or medication (e.g., fluoxetine) [9]. A similar significant association was found in another observational multicenter retrospective study conducted in patients with psychiatric disorders and hospitalized for severe COVID-19 [10]. A retrospective observational study also established a positive association between chronic administration of FIASMA and reduced mortality in COVID-19 hospitalized patients that was significant for the FIASMA amlodipine [11]. In a double-blind randomized clinical trial, outpatients treated with the FIASMA antidepressant fluvoxamine compared with placebo had a lower risk of clinical deterioration over 15 days of treatment [12]. The results of a prospective real-world evidence study also support this observation [13]. Finally, plasma markers of ceramide metabolism were found to be associated with respiratory severity and to correlate with inflammation in 49 patients hospitalized for COVID19 [14]. Taken together, these results show the potentially crucial importance of the ASM/ceramide system as a treatment target in COVID-19, a mechanism likely to be shared by all variants [15]. They also support the continuation of FIASMA medications during SARS-CoV-2 infection [9].

Second, anti-inflammatory properties of several antidepressants may have important value in regulating inflammation by inhibiting cytokine production in COVID-19. These anti-inflammatory effects might be explained (i) by the high affinity of certain antidepressants, such as fluvoxamine or fluoxetine, for Sigma-1 receptors [12, 16], which have been shown to restrict the endonuclease activity of an Endoplasmic Reticulum (ER) stress sensor called Inositol-Requiring Enzyme1 and to reduce cytokine expression without inhibiting classical inflammatory pathways, and/or (ii) by the inhibition of ASM in endothelial cells and the immune system $[6,7]$.

Finally, other potential mechanisms include reduction in platelet aggregation, decreased mast cell degranulation, interference with endolysosomal viral trafficking and increased melatonin levels [16].

These different but potentially interrelated mechanisms shared by several antidepressants such as fluvoxamine or fluoxetine, 
might collectively lead to anti-SARS-COV-2 effects while diminishing coagulopathy and cytokine storm consequences, which are known hallmarks of severe COVID-19.

Following these preclinical, observational and clinical converging findings, and as stated by Salles et al. [1] and Stip et al. [2], large-scale double-blind placebo-controlled randomized clinical trials of FIASMA antidepressants for COVID-19 at different stages of the disease, either alone or combined with medications that have shown preliminary evidence of potential efficacy and good tolerability, are urgently needed. Fluoxetine and fluvoxamine, which display high in vitro inhibition effect on ASM, showed potential positive effects at usual antidepressant doses, and are easy to use, including high safety margins, good tolerability, widespread availability and low cost, should be considered compelling treatments to prioritize for phase 3 trials against COVID-19 [9].

Nicolas Hoertel (D) ${ }^{1 凶}$, Marina Sánchez-Rico (1D ${ }^{1,2}$, Céline Cougoule ${ }^{3}$, Erich Gulbins ${ }^{4}$, Johannes Kornhuber ${ }^{5}{ }^{5}$, Alexander Carpinteiro ${ }^{4,6}$, Katrin Anne Becker ${ }^{4}$, Angela M. Reiersen (D ${ }^{7}$, Eric J. Lenze ${ }^{7}$, David Seftel ${ }^{8}$, Cédric Lemogne (iD ${ }^{9}$ and Frédéric Limosin ${ }^{1}$ ${ }^{1}$ DMU Psychiatrie et Addictologie, Département de Psychiatrie, Assistance Publique-Hôpitaux de Paris, Hôpital Corentin-Celton, INSERM, Institut de Psychiatrie et Neurosciences de Paris (IPNP), UMR_S1266, Université de Paris, Paris, France. ${ }^{2}$ Department of Psychobiology \& Behavioural Sciences Methods, Faculty of Psychology, Universidad Complutense de Madrid, Campus de Somosaguas, Pozuelo de Alarcón, Madrid, Spain. ${ }^{3}$ Institut de Pharmacologie et de Biologie Structurale, IPBS, Université de Toulouse, Toulouse, France. ${ }^{4}$ Institute of Molecular Biology, University Medicine Essen, University of Duisburg-Essen, Essen, Germany. ${ }^{5}$ Department of Psychiatry and Psychotherapy, University Hospital, Friedrich-Alexander University of Erlangen-Nuremberg, Erlangen, Germany. ${ }^{6}$ Department of Hematology and Stem Cell Transplantation, University Hospital Essen, University of Duisburg-Essen, Essen, Germany. ${ }^{7}$ Department of Psychiatry, Washington University in St. Louis School of Medicine, St. Louis, MO, USA. ${ }^{8}$ Golden Gate Fields Medical Clinic, Berkeley, CA, USA.

${ }^{9}$ Université de Paris, Assistance Publique-Hôpitaux de Paris, Hôpital Hôtel-Dieu, DMU Psychiatrie et Addictologie, Service de Psychiatrie de l'adulte, INSERM, Institut de Psychiatrie et Neurosciences de Paris (IPNP), UMR_S1266, Paris, France. ${ }^{\circledR}$ email: nico.hoertel@yahoo.fr

\section{REFERENCES}

1. Salles J, Briand-Mésange F, Trudel S, Ausseil J, Salles J-P, Chap H. Can antidepressants unlock prescription of rimonabant in the fight against COVID-19? Mol Psychiatry. 2021:1-2. https://doi.org/10.1038/s41380-021-01221-y. Epub ahead of print.

2. Stip E, Arnone D, Abdel Aziz K, Javaid S. Diversity of mechanism of action of psychotropic drugs in their anti-COVID-19 properties. Mol Psychiatry. 2021:1-5. https://doi.org/10.1038/s41380-021-01222-x. Epub ahead of print.

3. Hoertel N, Sánchez-Rico $M$, Vernet $R$, Beeker $N$, Jannot A-S, Neuraz $A$, et al. Association between antidepressant use and reduced risk of intubation or death in hospitalized patients with COVID-19: results from an observational study. Mol Psychiatry. 2021. https://doi.org/10.1038/s41380-021-01021-4 Epub ahead of print.

4. Hoertel N, Sánchez-Rico M, Vernet R, Beeker N, Jannot A-S, Neuraz A, et al. SSRIs and SNRIs and Risk of Death or Intubation in COVID-19: Results from an Observational Study. medRxiv 2020.07.09.20143339; https://doi.org/10.1101/ 2020.07.09.20143339.

5. Kornhuber J, Tripal $P$, Reichel $M$, Mühle $C$, Rhein $C$, Muehlbacher $M$, et al. Functional Inhibitors of Acid Sphingomyelinase (FIASMAs): a novel pharmacological group of drugs with broad clinical applications. Cell Physiol Biochem. 2010;26:9-20.

6. Carpinteiro A, Edwards MJ, Hoffmann M, Kochs G, Gripp B, Weigang S, et al. Pharmacological inhibition of acid sphingomyelinase prevents uptake of SARS-
CoV-2 by epithelial cells. Cell Rep Med. 2020;1:100142. https://doi.org/10.1016/j. xcrm.2020.100142. Epub 2020 Oct 29.

7. Carpinteiro A, Gripp B, Hoffmann M, Pöhlmann S, Hoertel N, Edwards MJ, et al. Inhibition of acid sphingomyelinase by ambroxol prevents SARS-CoV-2 entry into epithelial cells. J Biol Chem. 2021;296:100701. https://doi.org/10.1016/j. jbc.2021.100701. Epub 2021 Apr 23.

8. Schloer S, Brunotte L, Goretzko J, Mecate-Zambrano A, Korthals N, Gerke V, et al. Targeting the endolysosomal host-SARS-CoV-2 interface by clinically licensed functional inhibitors of acid sphingomyelinase (FIASMA) including the antidepressant fluoxetine. Emerg Microbes Infect. 2020;9:2245-55.

9. Hoertel N, Sánchez-Rico M, Gulbins E, Kornhuber J, Carpinteiro A, Lenze EJ, et al. Association between FIASMAs and Reduced Risk of Intubation or Death in Individuals Hospitalized for Severe COVID-19: an observational multicenter study. Clin Pharmacol Ther. 2021:10.1002/cpt.2317. https://doi.org/10.1002/cpt.2317. Epub ahead of print.

10. Hoertel N, Sánchez-Rico M, Gulbins E, Kornhuber J, Carpinteiro A, Abellán M, et al. Association between Psychotropic Medications Functionally Inhibiting Acid Sphingomyelinase and reduced risk of Intubation or Death among Individuals with Mental Disorder and Severe COVID-19: an Observational Study. medRxiv 2021.02.18.21251997; https://doi.org/10.1101/2021.02.18.21251997.

11. Darquennes G, Le Corre P, Le Moine O, Loas G. Association between functional inhibitors of acid sphingomyelinase (Fiasmas) and reduced risk of death in covid19 patients: a retrospective cohort study. Pharmaceuticals. 2021;14:226.

12. Lenze EJ, Mattar C, Zorumski CF, Stevens A, Schweiger J, Nicol GE, et al. Fluvoxamine vs Placebo and Clinical Deterioration in Outpatients With Symptomatic COVID-19: a Randomized Clinical Trial. JAMA. 2020;324:2292-2300.

13. Seftel D, Boulware DR. Prospective Cohort of Fluvoxamine for Early Treatment of Coronavirus Disease 19. Open Forum Infect Dis. 2021;8:ofab050.

14. Marín-Corral J, Rodríguez-Morató J, Gomez-Gomez A, Pascual-Guardia S, MuñozBermúdez R, Salazar-Degracia A, et al. Metabolic Signatures Associated with Severity in Hospitalized COVID-19 Patients. Int J Mol Sci. 2021;22:4794.

15. Fred SM, Kuivanen S, Ugurlu H, Casarotto PC, Levanov L, Saksela K, et al. Antidepressant and antipsychotic drugs reduce viral infection by SARS-CoV-2 and fluoxetine show antiviral activity against the novel variants in vitro. bioRxiv 2021.03.22.436379; https://doi.org/10.1101/2021.03.22.436379.

16. Sukhatme VP, Reiersen AM, Vayttaden SJ, Sukhatme VV. Fluvoxamine: a Review of Its Mechanism of Action and Its Role in COVID-19. Front Pharm. 2021;12:652688.

\section{AUTHOR CONTRIBUTIONS}

Writing-original draft: $\mathrm{NH}$; Writing-review \& editing: MS-R, CG, EG, JK, AC, KAB, $A M R, E J L, D S, C L, F L$.

\section{COMPETING INTERESTS}

$\mathrm{NH}, \mathrm{MS}-\mathrm{R}, \mathrm{EG}, \mathrm{JK}, \mathrm{AC}$, and $\mathrm{FL}$ are inventors on a patent application related to methods of treating COVID-19, filled by Assistance Publique - Hopitaux de Paris in France. NH has received personal fees and nonfinancial support from Lundbeck, outside the submitted work. AMR and EJL are inventors on a patent application related to methods of treating COVID-19, which was filed by Washington University in St. Louis. EJL has received consulting fees from Johnson and Johnson, and Jazz Pharmaceuticals. AMR has received grant or research support from the McDonnell Center for Systems Neuroscience, the McDonnell Center for Cellular and Molecular Neurobiology, and the Taylor Family Institute for Innovative Psychiatric Research. DS is the Chief Medical Officer and CEO of Enable Biosciences, a CLIA certified Federal clinical reference laboratory that performs COVID-19 antibody testing. CL reports personal fees and nonfinancial support from Janssen-Cilag, Lundbeck, Otsuka Pharmaceutical, and Boehringer Ingelheim, outside the submitted work. FL has received speaker and consulting fees from Janssen-Cilag, Euthérapie-Servier, and Lundbeck, outside the submitted work. Other authors declare no competing interests related to this work.

\section{ADDITIONAL INFORMATION}

Correspondence and requests for materials should be addressed to N.H.

Reprints and permission information is available at http://www.nature.com/ reprints

Publisher's note Springer Nature remains neutral with regard to jurisdictional claims in published maps and institutional affiliations. 